\title{
Synthesis of Pt nanoparticles and their burrowing into Si due to synergistic effects of ion beam energy losses
}

\author{
Pravin Kumar $^{* 1}$, Udai Bhan Singh ${ }^{1}$, Kedar Mal $^{1}$, Sunil Ojha ${ }^{1}$, Indra Sulania ${ }^{1}$, \\ Dinakar Kanjilal ${ }^{1}$, Dinesh Singh ${ }^{2}$ and Vidya Nand Singh ${ }^{2}$
}

\author{
Full Research Paper \\ Address: \\ ${ }^{1}$ Inter University Accelerator Centre (IUAC), New Delhi 110067, India \\ and ${ }^{2}$ National Physical Laboratory (NPL), New Delhi 110012, India \\ Email: \\ Pravin Kumar* - vishakhapk@gmail.com \\ * Corresponding author \\ Keywords: \\ atomic force microscopy; burrowing of nanoparticles; medium-energy \\ ion irradiation; nuclear and electronic energy loss; Rutherford \\ backscattering spectroscopy; scanning electron microscopy; thin \\ films; transmission electron microscopy
}

Beilstein J. Nanotechnol. 2014, 5, 1864-1872.

doi:10.3762/bjnano.5.197

Received: 09 May 2014

Accepted: 08 October 2014

Published: 24 October 2014

Associate Editor: A. Gölzhäuser

(C) 2014 Kumar et al; licensee Beilstein-Institut. License and terms: see end of document.

\begin{abstract}
We report the synthesis of Pt nanoparticles and their burrowing into silicon upon irradiation of a Pt-Si thin film with mediumenergy neon ions at constant fluence $\left(1.0 \times 10^{17} \mathrm{ions} / \mathrm{cm}^{2}\right)$. Several values of medium-energy neon ions were chosen in order to vary the ratio of the electronic energy loss to the nuclear energy loss $\left(S_{\mathrm{e}} / S_{\mathrm{n}}\right)$ from 1 to 10 . The irradiated films were characterized using Rutherford backscattering spectroscopy (RBS), atomic force microscopy (AFM), scanning electron microscopy (SEM), X-ray diffraction (XRD) and high resolution transmission electron microscopy (HRTEM). A TEM image of a cross section of the film irradiated with $S_{\mathrm{e}} / S_{\mathrm{n}}=1$ shows $\approx 5 \mathrm{~nm}$ Pt NPs were buried up to $\approx 240 \mathrm{~nm}$ into the silicon. No silicide phase was detected in the XRD pattern of the film irradiated at the highest value of $S_{\mathrm{e}} / S_{\mathrm{n}}$. The synergistic effect of the energy losses of the ion beam (molten zones are produced by $S_{\mathrm{e}}$, and sputtering and local defects are produced by $S_{\mathrm{n}}$ ) leading to the synthesis and burrowing of $\mathrm{Pt} \mathrm{NPs}$ is evidenced. The Pt NP synthesis mechanism and their burrowing into the silicon is discussed in detail.
\end{abstract}

\section{Introduction}

The emergence of nanotechnology has opened up new research channels in almost every field of science [1-8]. The synthesis of nano-dimensional structures of various elements with narrow size distribution is a big challenge for scientists [9-11]. Due to certain advantages, namely, the control of growth parameters and spatial distribution, ion beam synthesis of buried nanoparticles (NPs) has received considerable attention in recent years [12-15]. The ions of desired elements, especially those of noble metals, are implanted into a matrix with certain fluence and post-annealing of the sample leads to the formation of NPs 
within the matrix. The energy, which governs the stopping of ions in matter, is chosen to obtain a desired particle distribution profile (longitudinal) in the matrix. The multiple energy implantations of the ions are used to increase this distribution profile further [16]. The transverse distribution is controlled by scanning the ion beam over the sample (desired matrix). The ion fluence and the annealing temperature are chosen to control the growth process leading to the final size distribution of the particles [17]. Homogenous nucleation requires a threshold concentration of implanted materials. Further, annealing may affect the spatial distribution of particles significantly due to thermally activated diffusion of implants.

Due to the fact that metallic ion beams produced from the electron cyclotron resonance ion source (ECRIS) (used in the current experiment) suffer from poor intensities and instabilities [18,19], a recently investigated novel method of synthesizing buried metallic NPs has been employed [20]. The ion irradiation of thin metallic films deposited on a suitable substrate (with lower surface energy) leads to the synthesis of metal NPs embedded into the substrate. The energy losses of the ions (not the ion itself) are mainly responsible for the resulting nanostructuring. The ion-induced point defects lead to the burrowing/diffusion of the surface NPs. The depth of the defect cascade can be more than the range. Only a few reports of such studies are available [21-26] and the exact mechanism of the formation of NPs is not very clear. The burrowing of self-organized cobalt clusters in a gold substrate upon thermal activation was reported by Padovani et al. [27]. When the surface energy of the metallic film is larger than that of the substrate, then surface nano-structuring is due to ion-induced sputtering of the film followed by the dewetting of metallic islands [20,28]. However, other effects such as ion-induced viscous flow, recoil implantation and thermodynamically driven capillary forces can also contribute to the formation of the buried NPs. When the ion beams with high electronic energy loss (dominates at high energies) pass through the material, a local melting (thermal spike) [29] occurs along the ion trajectory due to the energy deposition into the electronic subsystem (within $10^{-16} \mathrm{~s}$ ). The local thermalization of the electronic sub-system takes place within $10^{-14} \mathrm{~s}$. The deposited energy is transferred to the atomic subsystem by electron-phonon coupling. The melting of materials along the ion trajectory generates a surface tension gradient due to an imbalance of the surface and the interface energies, which further gives rise to mass transport through capillary action. The migration of metallic atoms and subsequent agglomeration can result in the formation of the nanoparticles. The ion trajectory formation in insulators and semiconductors after passage of high energy ions is mainly explained by the Coulomb explosion model [30]. However, ion beams with high nuclear energy loss (which dominates at low energies) in the materials undergo elastic scattering with the atoms of materials (for instance Pt and $\mathrm{Si}$ as in the present case), and finally, a collision cascade is achieved. Bolse [31] reported that a cylindrical local spike can be formed along a sub-cascade by the overlap of spherical thermal spikes.

To decouple the ion-matter interactions in the two types of energy loss processes and to better understand the synthesis mechanism of the NPs and their burrowing, neon ions of several energies were chosen. The interest in choosing Pt as the thin film was due to potential applications of Pt NPs [32-34]. Apart from their excellent catalytic performance, Pt NPs are used in fabricating super capacitors [35]. The Pt NPs in core-shell structures (Pt forms the shell) are used in surface enhanced Raman scattering (SERS) studies [36] as well. Moreover, Pt is relatively inert in atmosphere and ex situ characterization of irradiated samples can also be carried out. In this paper, we present the synthesis of Pt NPs and their burrowing in Si and discuss the possible mechanism.

\section{Experimental}

Using thermal evaporation (deposition rate, $0.1 \mathrm{~nm} / \mathrm{s}$ ) under high vacuum conditions, $5 \mathrm{~nm} \mathrm{Pt}$ thin films were deposited on a crystalline silicon substrate. The pressure inside the chamber before and during deposition was $2 \times 10^{-7}$ and $3 \times 10^{-6}$ mbar, respectively. The samples $(\mathrm{Pt}-\mathrm{Si}, 10 \times 10 \mathrm{~mm})$ were irradiated using an ECRIS-based, upgraded version of the old low energy ion beam facility (LEIBF) [37] at IUAC, New Delhi. Ion irradiation was carried out in a vacuum chamber $\left(\approx 10^{-7}\right.$ mbar pressure) at normal incidence and at room temperature. All the samples were processed at an ion fluence of $1 \times 10^{17}$ ions $/ \mathrm{cm}^{2}$ with a constant beam current of $1 \mu \mathrm{A}$. The ion beam was scanned over the $15 \times 15 \mathrm{~mm}$ area to achieve uniform irradiation conditions. The chosen beam energies for irradiation were $50,140,350$ and $600 \mathrm{keV}$. For $350 \mathrm{keV}$ and $600 \mathrm{keV}$ ion irradiations, $\mathrm{Ne}^{+2}$ and $\mathrm{Ne}^{+3}$ ions were extracted from the ECR plasma and $E / q$ values (the total potential difference including extraction and platform voltages) were set to $175 \mathrm{kV}$ and $200 \mathrm{kV}$, respectively. The extraction of highly charged ions was employed to meet the energy requirements as accelerator operation with platform voltage beyond $250 \mathrm{kV}$ was quite unsafe. Singly ionized neon ions were extracted for the irradiation of the films at other two energies and $E / q$ values $(50 \mathrm{kV}$ for $50 \mathrm{keV}$, and $140 \mathrm{kV}$ for $140 \mathrm{keV}$ ) were set accordingly. The irradiated samples were characterized using Rutherford backscattering spectroscopy (RBS), atomic force microscopy (AFM), scanning electron microscopy (SEM) and X-ray diffraction (XRD) techniques. High resolution cross sectional transmission electron microscopy (HRXTEM) of the sample irradiated with $S_{\mathrm{e}} / S_{\mathrm{n}}=1$ (where maximum burrowing was seen) was also performed in order to gain quantitative information, for 
example, particle size, depth of burrowing, etc. The morphological changes on the surfaces were studied using a multimode Nanoscope IIIa atomic force microscopy (AFM) in tapping mode. The AFM scans were made at a slower rate using a single crystal silicon tip. The apex of the tip has a radius of curvature of $\approx 10 \mathrm{~nm}$ and a locking frequency $\approx 350 \mathrm{KHz}$. For RBS measurements, $2 \mathrm{MeV} \mathrm{He}{ }^{+}$ions were bombarded onto the samples using the Pelletron Accelerator RBS-AMS System (PARAS) facility at IUAC, New Delhi. The backscattering yield was measured using a surface barrier detector mounted at $10^{\circ}$ in the irradiation chamber with respect to the beam direction. The vacuum inside the chamber during irradiation was $\approx 10^{-4}$ Torr. $\mathrm{He}^{+}$irradiation was carried out at $7^{\circ}$ to avoid ion channeling in the samples. Before taking the spectra, an energy calibration was performed using the Au and Si edges (reference sample: Au deposited on the glass). For HRXTEM analysis, the sample was cut in $4 \times 5 \mathrm{~mm}$ pieces using an ultrasonic disc cutter. These pieces were glued together (face-to-face and faceto-back) to form a cross. A $2.3 \mathrm{~mm}$-diameter piece was drilled out (along the cross section) using an ultrasonic cutter. This piece was fixed (using epoxy) in a $3 \mathrm{~mm}$-diameter brass tube. Thin slices were cut from this tube for mechanical thinning up to $100 \mu \mathrm{m}$. Then, the center of the slice was dimpled to achieve 20-30 $\mu \mathrm{m}$ thickness. The dimpled slice was ion milled to achieve final perforation and TEM analysis was performed. An $\mathrm{X}$-ray diffractometer installed at IUAC was equipped with a conventional $\mathrm{Cu} \mathrm{K}_{\alpha}$ source, Göbble mirror, LiF monochromator, scintillator detector $(\mathrm{NaI}(\mathrm{Tl}))$ and was used to record the XRD pattern of pristine and irradiated films. For SEM measurements, a field emission scanning electron microscope (FE-SEM, MIRA II LMH from TESCAN) installed at IUAC with a resolution of $1.5 \mathrm{~nm}$ at $30 \mathrm{kV}$ was used. This model has a secondary electron (SE) and a backscattered electron (BSE) detector for imaging.

\section{Results and Discussion}

Using stopping and range of ions in matter (SRIM) calculations [38], the energy losses (both electronic and nuclear) by neon ions in the Pt film as a function of ion energy is shown in Figure 1.

Unlike swift, heavy ions (with an energy of approximately hundreds of $\mathrm{MeV}$ ) that undergo very high (on the order of $\mathrm{keV} / \AA \AA)$ electronic energy loss $\left(S_{\mathrm{e}}\right)$ in the material, $S_{\mathrm{e}}$ by neon ions of chosen energies in Pt is quite low $(\approx 155 \mathrm{eV} / \AA$ for $600 \mathrm{keV})$. For $50 \mathrm{keV}$ neon ions, electronic $\left(S_{\mathrm{e}}\right)$ and nuclear stopping $\left(S_{\mathrm{n}}\right)$ are quite close to each other $(\approx 30 \mathrm{eV} / \AA)$. With increasing energy, $S_{\mathrm{e}}$ increases and $S_{\mathrm{n}}$ decreases ( $y$ axis on the left hand side). The energy loss ratio, $S_{\mathrm{e}} / S_{\mathrm{n}}$, is also plotted as a function of ion energy for convenience ( $y$ axis on the right hand side).

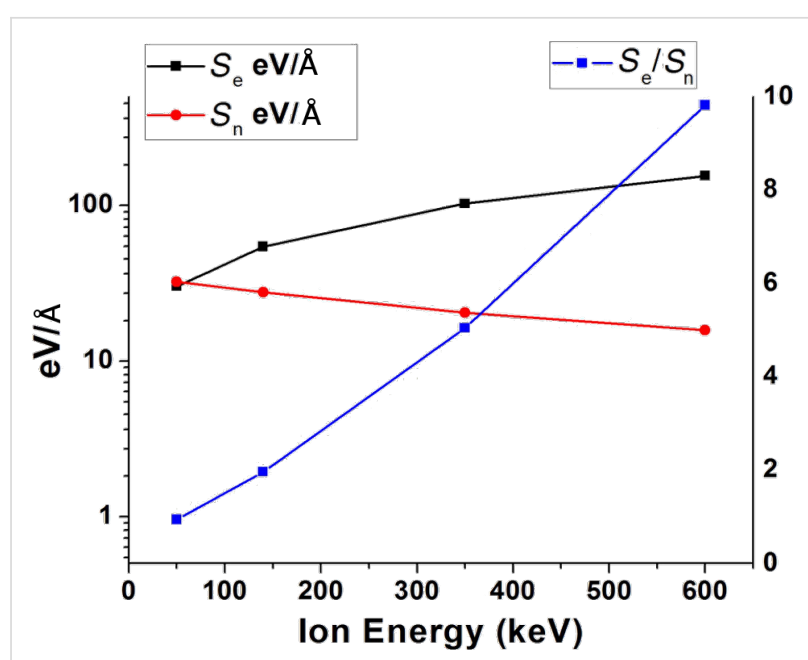

Figure 1: Electronic and nuclear stopping vs ion energy (SRIM calculation for neon ions incident on $\mathrm{Pt}$ ).

The 2D surface morphology along with the sectional analysis (shown in the right hand side) of the pristine and ion-irradiated films deduced by AFM is shown in Figure 2a-e.

In high-energy irradiated samples (Figure 2d,e) where $S_{\mathrm{e}}$ dominates, the appearance of uniform structures on the surface seems to be due to dewetting of Pt films. Since the kinetic sputtering of the film (dominated by high $S_{\mathrm{n}}$ ) is less in these two samples, well-isolated Pt islands are not visible on the surface. The large height variation on the surface as seen in the sectional analysis of the respective AFM images is due to Pt agglomeration (confirmed by the energy dispersive X-ray analysis) on the surface after ion irradiation. The sputtering of $\mathrm{Pt}$ and its re-deposition on the surface cannot result in such a uniform pattern on the surface. Therefore, transient thermal effects, activated by dewetting, are assumed to yield the uniform surface structures. These surface structures begin to disappear as the $S_{\mathrm{e}}$ decreases (see Figure 2c). In the sample irradiated with $50 \mathrm{keV}$ $\left(S_{\mathrm{e}} / S_{\mathrm{n}}=1\right)$, the surface structures disappear completely.

Figure 3 shows the Rutherford backscattering spectra (at the Pt edge) of pristine and irradiated samples. The shifting of the $\mathrm{Pt}$ peak towards lower energy with a decrease in the $S_{\mathrm{e}} / S_{\mathrm{n}}$ ratio confirms the burrowing of Pt in Si. The Si edge (not shown here), however, remains unshifted due to the fact that energy loss by $\mathrm{He}^{+}$ions in $5 \mathrm{~nm}$ thin $\mathrm{Pt}$ is negligible. The reduction in the height of the peak with decreasing $S_{\mathrm{e}} / S_{\mathrm{n}}$ shows the ioninduced sputtering of Pt. About a 50\% Pt loss (area under the curve) is estimated in the film irradiated with $50 \mathrm{keV}$ neon ions.

The Pt peaks for the different irradiation conditions are not fully resolved due to the extremely small thickness of the films. By taking the energy difference of the pristine Pt peak and the irra- 

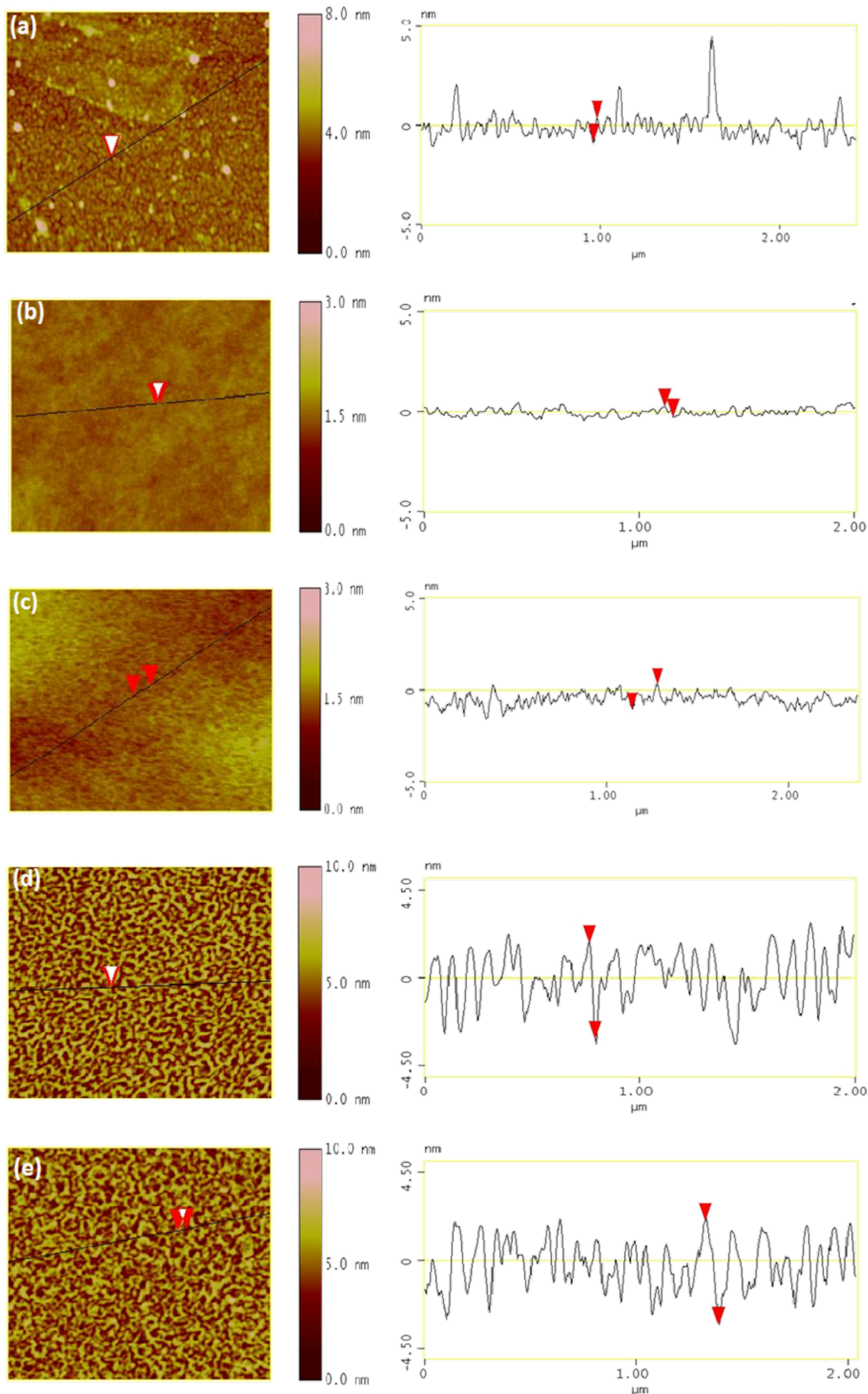

Figure 2: AFM images: a) pristine film, b-e) films irradiated with $50 \mathrm{keV}, 140 \mathrm{keV}, 350 \mathrm{keV}$ and $600 \mathrm{keV}$, respectively. 


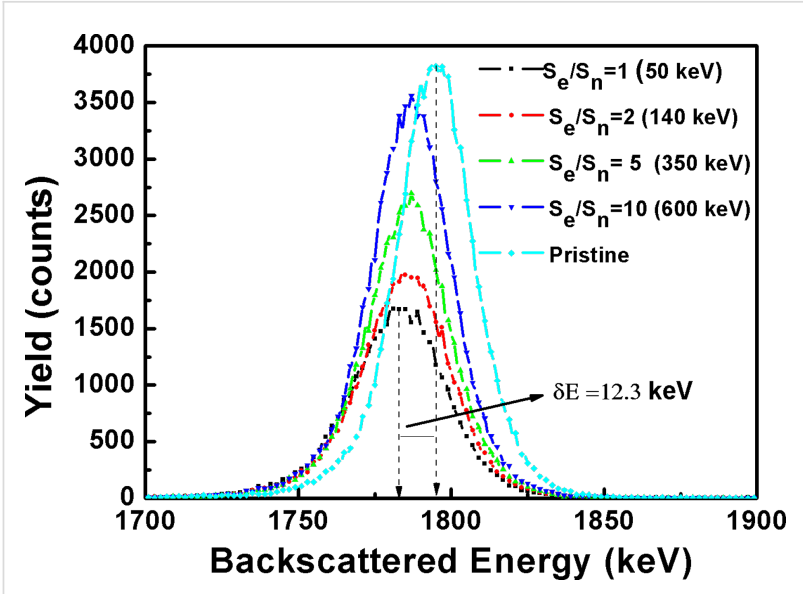

Figure 3: Rutherford backscattering spectra of the pristine and the irradiated films (Pt-Si).

diated $\left(S_{\mathrm{e}} / S_{\mathrm{n}}=1\right)$ film $(12.3 \mathrm{keV})$, and utilizing the energy loss of the helium ions in the Pt-Si system as a function of the depth, $\approx 2 \mathrm{~nm}$ of burrowing was calculated in the irradiated film. If the elemental concentration is less than $10^{14}$ ions $/ \mathrm{cm}^{2}$, detection with conventional RBS is difficult. In such a case, highresolution, highly sensitive RBS measurements would be needed for accurate quantitative information on the burrowing.

The SEM images of the pristine and the ion-irradiated samples (only for $350 \mathrm{keV}$ and $600 \mathrm{keV}$ ) are shown in Figure $4 \mathrm{a}-\mathrm{c}$. For the other samples irradiated with $140 \mathrm{keV}$ and $50 \mathrm{keV}$ energies, we could not get good contrast on the surface. As shown in Figure $4 \mathrm{a}$, the $\mathrm{Pt}$ film on the Si is not very uniform. The formation of NPs $(\approx 20 \mathrm{~nm}$ white spots in Figure $4 \mathrm{c}$ and $<20 \mathrm{~nm}$ in Figure $4 \mathrm{~b}$ ) on the surface upon ion irradiation is confirmed. However, fade contrast in Figure 4b, in the sample irradiated with $350 \mathrm{keV}$, can either be due to the partial sinking of NPs in the substrate or due to the reduction in the size of the particles.
SEM gives elemental information on the surface while AFM provides surface topography. Therefore, the features in the SEM and the AFM images cannot be compared quantitatively. Moreover, it is not possible to pinpoint the exact surface area by taking images with these two techniques. If surface structures are uniform, the correlation between the features governed by AFM and SEM can be discussed qualitatively. The Pt islands formed by kinetic sputtering followed by possible dewetting as seen in the AFM images (Figure $2 \mathrm{~d}$ and $2 \mathrm{e}$ ) are visible in the SEM images (Figure $4 \mathrm{~b}$ and $4 \mathrm{c}$ ) as white spots. The energy dispersive X-ray analysis shows a relatively larger atomic fraction of $\mathrm{Pt}$ when the electron beam is focused on these white spots. The discontinuous Pt film (pristine sample) as seen in the SEM image (Figure 4a) is also visible in the AFM image (Figure 2a) with certain grain size. The unusual heights at certain positions in the image (Figure 2a) may be due to dust particles on the surface.

The high resolution XTEM analysis of one sample (irradiated with $S_{\mathrm{e}} / S_{\mathrm{n}}=1$ ) is shown in Figure 5. Figure 5a represents the cross sectional view of two silicon surfaces (cut from the same sample) joined face-to-face with glue (epoxy/adhesive substance). The range of $50 \mathrm{keV}$ neon ions in $\mathrm{Si}$ is $\approx 107 \mathrm{~nm}$ with a longitudinal straggling of $\approx 46 \mathrm{~nm}$. Therefore, one can expect a modified region of $\approx 130 \mathrm{~nm}$ below the surface upon ion bombardment. However, an interface at $\approx 242 \mathrm{~nm}$ below the surface is clearly seen in Figure 5a. This is mainly attributed to the amorphization of the silicon by collision cascade which can propagate even further than the range of ions (see Si vacancies profile distribution in Figure 6). Figure 5b, which is the zoomed image of the region covering the surface and the interface caused by the cascade (arrow moves from the surface to interface), shows that NPs are present up to the end of the collision cascade. However, the density of NPs decreases drastically with increasing depth (which could be less than the detection limit of
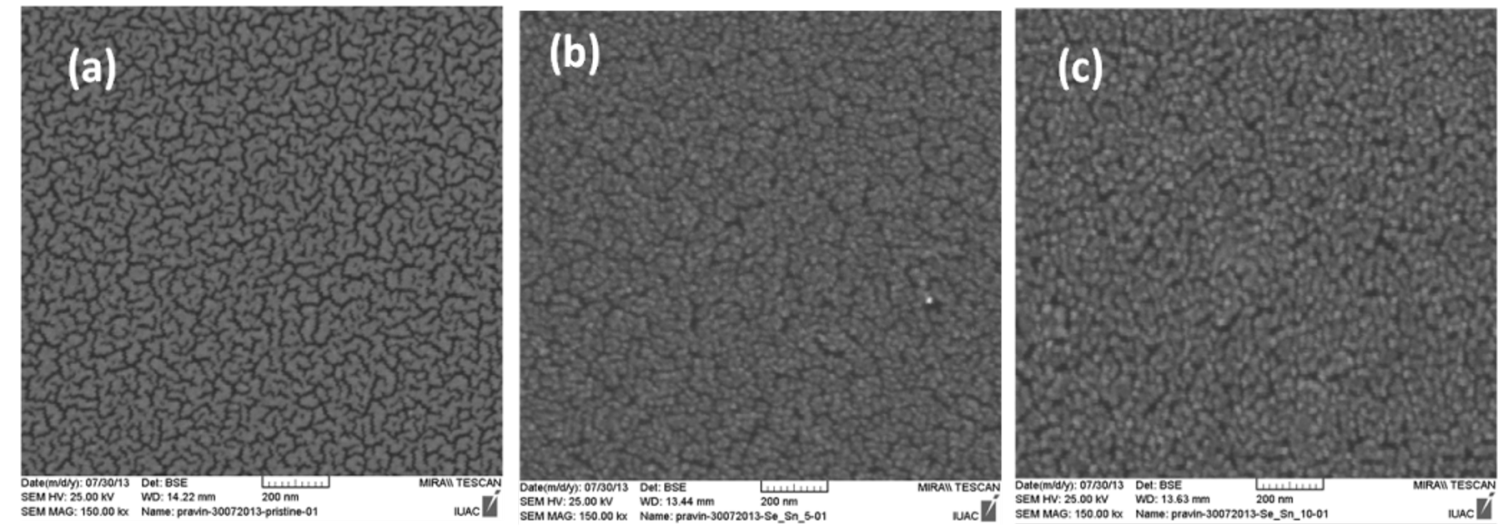

Figure 4: SEM images: a) pristine sample, b) $350 \mathrm{keV}$ ion-irradiated film, and c) $600 \mathrm{keV}$ ion-irradiated film. 


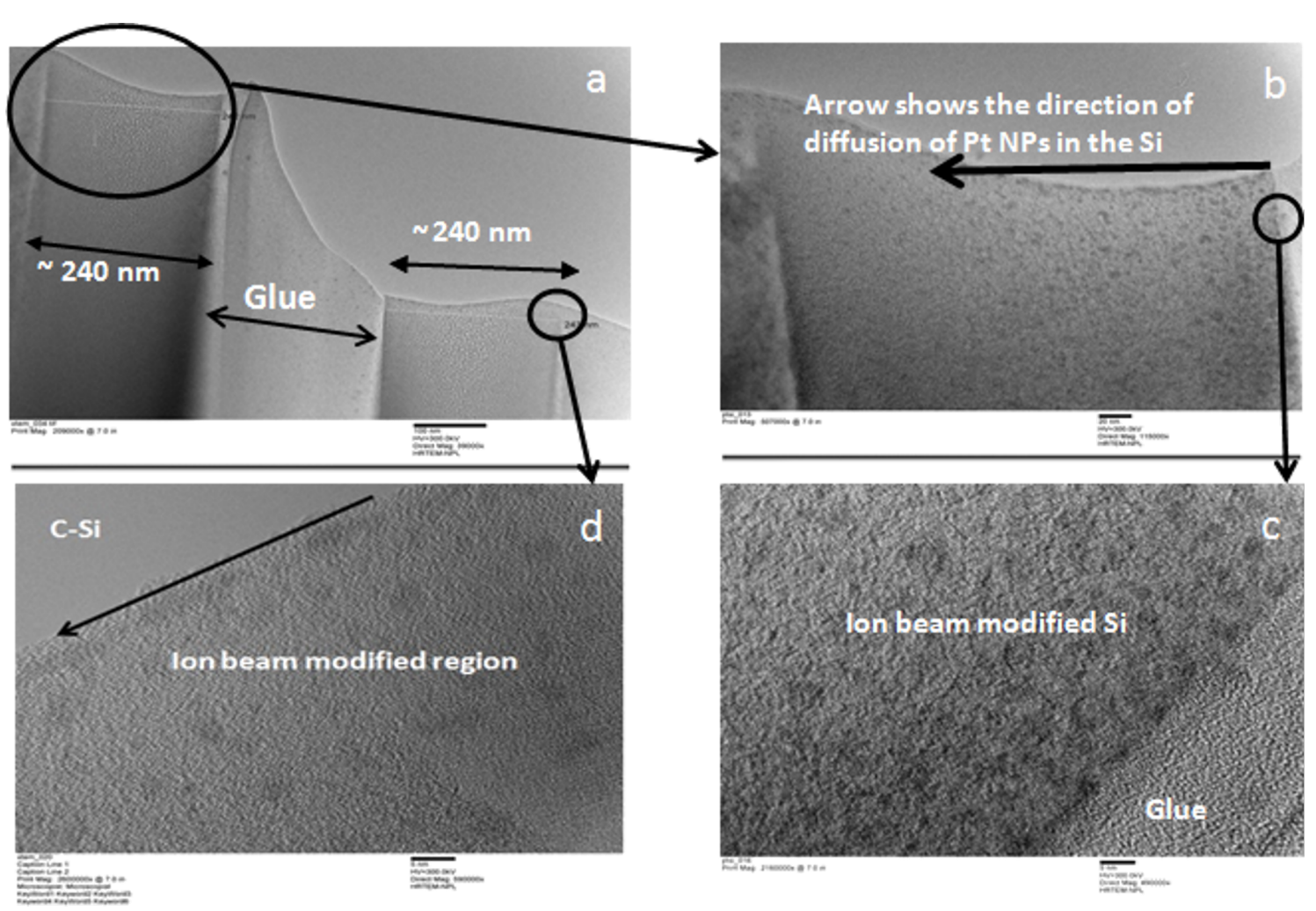

Figure 5: TEM images a) various interfaces, b) density distribution of NPs in ion beam modified region, c) interface showing high density of NPs near surface and absence of Pt on the surface, and d) NP distribution near the end of collision cascade.

the RBS within a few $\mathrm{nm}$ ) and this is probably the reason why the Pt edge has a small shift (compared to pristine) in the RBS measurement of the sample. Figure $5 \mathrm{c}$ covers the near-surface region of HRTEM analysis which shows that $\approx 5 \mathrm{~nm}$ crystalline NPs are uniformly distributed below the surface. The density and the size distribution of NPs close to the end of collision cascade are shown in Figure 5d. The density of NPs decreases drastically. The size of NPs does not change much.

When varying the $S_{\mathrm{e}} / S_{\mathrm{n}}$ ratio, the maximum burrowing of $\mathrm{Pt}$ NPs was found for the films irradiated with $50 \mathrm{keV}$ neon ions. Therefore, local defects (especially vacancies) produced by elastic collisions, which are governed by $S_{\mathrm{n}}$, are mainly responsible for the burrowing of NPs in silicon as also discussed by $\mathrm{Hu}$ et al. [22]. Given the irradiation parameters (50 keV energy, $1 \mu \mathrm{A}$ beam current and $16 \times 10^{3} \mathrm{~s}$ to irradiate $10^{17} \mathrm{ions} / \mathrm{cm}^{2}$ in a sample of $1 \mathrm{~cm}^{2}$ area) and the specific heat of silicon $(710 \mathrm{~J} / \mathrm{kg} \cdot \mathrm{K})$, we expected the target temperature to be at $\approx 500 \mathrm{~K}$ at the end of the irradiation [39]. The radiation and the heat conduction losses were not considered in the calculation. The diffusivity $(D)$ of $\mathrm{Pt}$ in silicon at $500 \mathrm{~K}$ is $\approx 3 \times 10^{-19} \mathrm{~m}^{2} / \mathrm{s}$ [40]. The total energy deposited $\left(F_{\mathrm{D}}\right)$ by $50 \mathrm{keV}$ ions in $\mathrm{Si}$ is $\approx 38 \mathrm{eV} / \AA$. Given a target temperature of $500 \mathrm{~K}$ $\left(k_{\mathrm{B}} T=41 \mathrm{meV}\right)$, an ion irradiation time, $t$, of $16 \times 10^{3} \mathrm{~s}$, and the

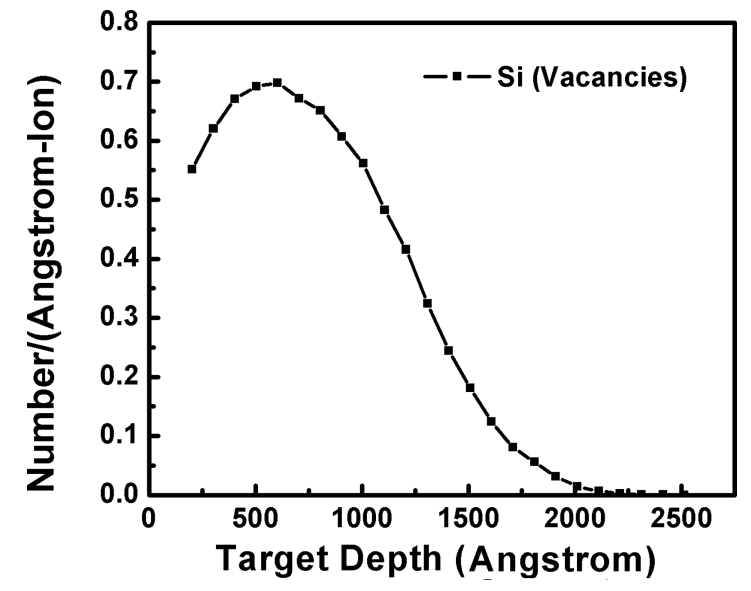

Figure 6: The distribution of silicon vacancies. The $50 \mathrm{keV}$ neon ions were irradiated at normal incidence on $5 \mathrm{~nm}$ Pt film deposited on silicon substrate.

relation $d=D \times k_{\mathrm{B}} T \times t / F_{\mathrm{D}}$ (where $k_{\mathrm{B}}$ is the Boltzmann constant), the diffusion length (d) of Pt into crystalline silicon is estimated to be $\approx 50 \mathrm{~nm}$. Therefore, the presence of NPs beneath the surface and up to $\approx 250 \mathrm{~nm}$ is probably due to radiationinduced enhanced diffusion. Holm et al. [41] have reported Pt 
distribution into lightly damaged regions of silicon approximately congruous to the vacancies generated during implantation. At a typical fluence of $10^{11}$ ions $/ \mathrm{cm}^{2}$, they observed an enhanced Pt accumulation approximately two orders of magnitude higher compared to diffusion in non-implanted silicon. At the very high fluence $\left(10^{17}\right.$ ions $\left./ \mathrm{cm}^{2}\right)$ in the present study, large vacancies produced by elastic collisions between ions and target atoms can give rise to an enhanced diffusion of $\mathrm{Pt}$ via a Frank-Turnbull mechanism [42], which requires a relatively low processing temperature. In Frank-Turnbull-type diffusion, impurity atoms/clusters ( $\mathrm{Pt}$ in this case) move from the interstitial sites to the vacancies. The vacancy production in the materials during ion irradiation/implantation is linearly proportional to the ion fluence [43]. Therefore, enhanced diffusion of Pt via vacancy production by the ion irradiation at high fluence is quite possible. Furthermore, the silicon vacancy profile (TRIM calculation/simulation; shown in Figure 6) upon $50 \mathrm{keV}$ neon ion irradiation, which seems to be responsible for the $\mathrm{Pt}$ diffusion, matches well with the NP distribution (obtained from the cross sectional HRTEM analysis) in the film irradiated under the same conditions. Total vacancies produced in the system for a chosen ion-target combination is $846 /$ ion (TRIM calculations). Using the thermal properties of silicon (a specific heat of $710 \mathrm{~J} / \mathrm{kg} \cdot \mathrm{K}$ and a thermal conductivity of $150 \mathrm{~W} / \mathrm{m} \cdot \mathrm{K}$ ) and electronic energy deposited by the ions in silicon, we expect a spike temperature of about $\approx 2540 \mathrm{~K}$ (within 1 ps and 1 $\mathrm{nm}$ away from the ion track) [44]. The melting point of silicon is $\approx 1400 \mathrm{~K}$ and transient molten zones (giving rise to viscous flow of Pt atoms) in silicon are possible by ion irradiation. Since the temperature spike quenches via electron-phonon coupling within $10^{-11} \mathrm{~s}$, a very small contribution by the viscous flow in Pt diffusion is expected for entire irradiation time.

Figure 7 shows the distribution of Pt recoils (TRIM calculation/ simulation) for $50 \mathrm{keV}$ neon ion irradiation on the Pt-Si system at normal incidence. The TRIM calculation takes an unperturbed system (point defects created by preceding ions are ignored) into account for each incident ion. The distribution shows that $\mathrm{Pt}$ atoms undergo near-surface recoil implantation upon ion irradiation.

By observation of the uniform size of the Pt NPs (up to $240 \mathrm{~nm}$ beneath the surface), the density distribution of NPs (density decreases from surface to bulk), and the recoil distribution profile, it seems that the synthesis of the NPs takes place near the surface. The Pt NPs may then diffuse into the silicon via vacancies. Yet the question of the possible Pt NP formation mechanism for this experiment still remains. Considering the AFM and RBS measurement results (energy dependent changes in surface topography, shift and intensity loss in Pt peaks), we

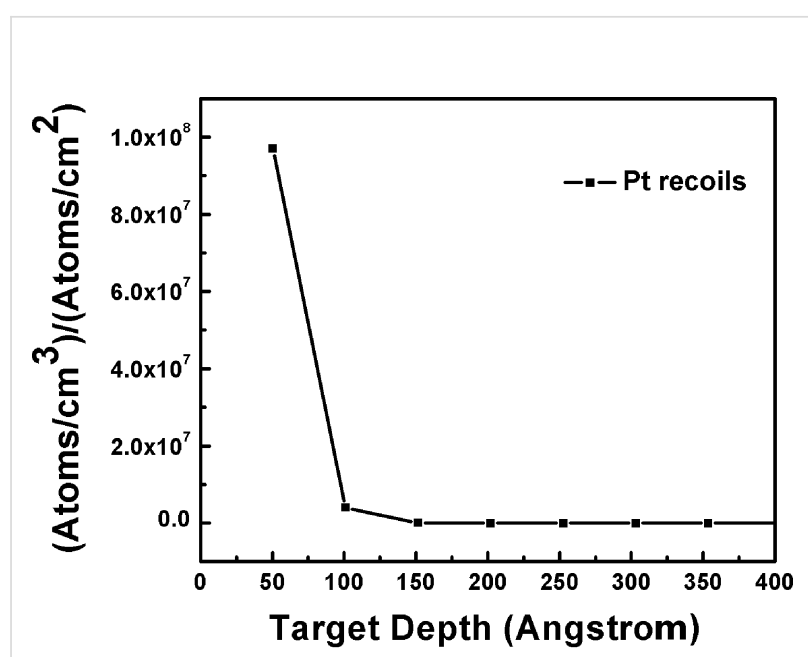

Figure 7: The distribution of $\mathrm{Pt}$ recoils $\left(\mathrm{Pt} / \mathrm{cm}^{3}\right.$ per $\left.\mathrm{Ne} / \mathrm{cm}^{2}\right)$. The $50 \mathrm{keV}$ neon ions were irradiated at normal incidence on $5 \mathrm{~nm}$ Pt film deposited on the silicon substrate.

believe that the nuclear sputtering of the Pt film takes place during ion irradiation resulting in Pt islands on the Si surface. Transient thermal spikes generated by the ion beams are sufficient enough to melt the Pt islands. The spike temperature in $\mathrm{Pt}$ is expected to be $\approx 2000 \mathrm{~K}$ (within $10^{-14} \mathrm{~s}$ and $1 \mathrm{~nm}$ away from the ion trajectory) upon irradiation with $50 \mathrm{keV}$ neon ions. In comparison to silicon, the temperature rise in $\mathrm{Pt}$ is faster due to its high electron density. The spike temperature in Pt is sufficient for the transient melting of islands (melting point of $\mathrm{Pt}$ is $\approx 1768 \mathrm{~K}$ ). The molten Pt islands take a spherical shape to minimize their surface energy (dewetting). The surface energies of $\mathrm{Pt}$ and silicon are 2.49 and $1.51 \mathrm{~J} / \mathrm{m}^{2}$, respectively. There could be electronic sputtering of these molten $\mathrm{Pt}$ islands giving rise to a uniform size of NPs on the surface. The recoiled Pt atoms underneath the surface may also agglomerate during thermal spikes giving rise to the satellite Pt NPs as reported in the literature $[22,45]$. The Ostwald ripening [46] of these satellite NPs can result in the final size $(\approx 5 \mathrm{~nm}$ as seen by HRXTEM analysis) of NPs which undergo diffusion into the silicon. In Ostwald ripening, bigger clusters are grown at the expense of the dissolution of smaller cluster. The necessary temperature for this process is achieved through energy deposition from the ion beam. To confirm the formation of a silicide phase (if any) in irradiated films, the XRD patterns of pristine and ion-irradiated (only with $S_{\mathrm{e}} / S_{\mathrm{n}}=10$ ) films were recorded and are shown in Figure 8 . The film irradiated with a high $S_{\mathrm{e}}$ is expected to undergo a phase formation due to a higher spike temperature along with the mingling of atoms by elastic collisions. Within the diffractometer detection limit, we could not find any silicide phase in the irradiated film and we believe that NPs (reported for film irradiated with $S_{\mathrm{e}} / S_{\mathrm{n}}=1$ ) consist only of Pt. 
We also expect absence of a silicide phase in the films irradiated with lower $S_{\mathrm{e}}$. The Pt film is polycrystalline in nature and (111) and (200) planes are clearly visible in the XRD pattern [47]. The reduced intensities and the broadening of the Pt peaks in the irradiated film confirm the Pt loss on the surface and the formation of NPs. The peak at around $2 \theta=56^{\circ}$ in the irradiated film is due to the Si substrate. The sharp feature just before $\mathrm{Si}$ substrate peak is a non-Bragg scattering peak.

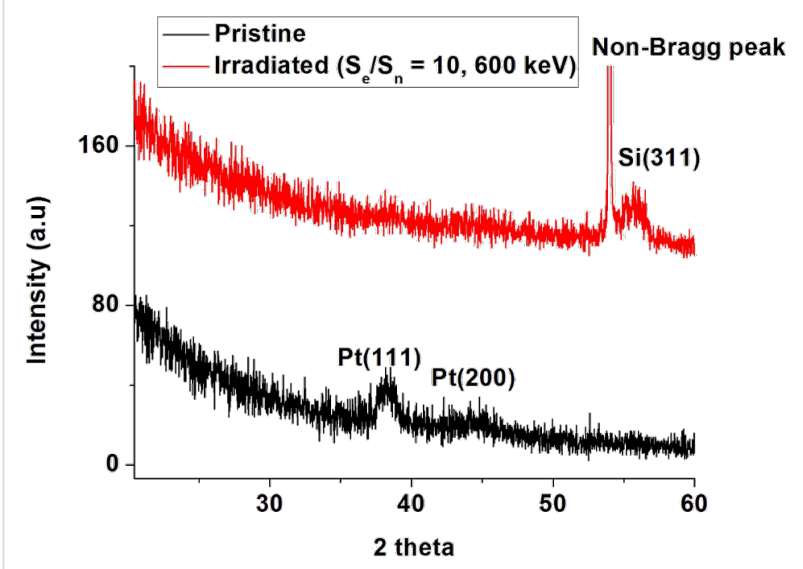

Figure 8: The XRD patterns of the pristine and the ion irradiated $\left(S_{\mathrm{e}} / S_{\mathrm{n}}=10\right)$ films.

\section{Conclusion}

We have reported the synthesis and the burrowing of Pt NPs due to medium-energy neon ion irradiation for Pt thin films deposited on a silicon substrate $(\mathrm{Pt}-\mathrm{Si})$. The ion fluence was kept constant $\left(10^{17}\right.$ ions $\left./ \mathrm{cm}^{2}\right)$ during the irradiation. Several ion energies (50 keV, $140 \mathrm{keV}, 350 \mathrm{keV}$ and $600 \mathrm{keV}$ ) were chosen to vary $S_{\mathrm{e}} / S_{\mathrm{n}}(1,2,5$ and 10$)$ in the Pt-Si system. The synthesis of Pt NPs and their burrowing in Si was confirmed using AFM, SEM, XRD and RBS measurements. The relation between the energy losses of the ion beam and the synthesis and burrowing of Pt NPs are evidenced. The HRXTEM analysis of a single sample (irradiated with $S_{\mathrm{e}} / S_{\mathrm{n}}=1$ ) shows that the size of the NPs and the depth of the burrowing are $\approx 5 \mathrm{~nm}$ and $\approx 240 \mathrm{~nm}$, respectively. Regarding the depth of the burrowing, the density of the NPs decreases drastically. The XRD analysis shows an absence of silicide phase within the detection limit of the instrument. Ion beam induced sputtering followed by partial dewetting of metallic films and recoil implantation seems to be the possible mechanism behind Pt NP $(\approx 5 \mathrm{~nm})$ formation. The ioninduced, silicon vacancy profile matches well with the Pt NP distribution underneath the surface. Therefore, radiation enhanced diffusion, in particular a Frank-Turnbull-type mechanism, is likely responsible for the large diffusion $(\approx 240 \mathrm{~nm}$ deep) of Pt NPs into the silicon.

\section{Supporting Information}

\section{Supporting Information File 1}

Additional information.

[http://www.beilstein-journals.org/bjnano/content/

supplementary/2190-4286-5-197-S1.pdf]

\section{Acknowledgements}

One of the authors (PK) thanks Dr. D. K. Avasthi for extending the XRD, AFM and SEM facilities to characterize the films. Help received from Mr. Subodh Gautam for XRD measurements and Mr. Saif Ahmed Khan for SEM measurements is highly acknowledged. PK also thanks the colleagues in the target lab for the thin film deposition. The discussions with Dr. F. Singh were very useful in preparing the manuscript. Special thanks are due to Dr. Sadhana Singh for moral support.

\section{References}

1. Urdampilleta, M.; Klyatskaya, S.; Cleuziou, J.-P.; Ruben, M.; Wernsdorfer, W. Nat. Mater. 2011, 10, 502. doi:10.1038/nmat3050

2. Tang, J.; Huo, Z.; Brittman, S.; Gao, H.; Yang, P. Nat. Nanotechnol. 2011, 6, 568-572. doi:10.1038/nnano.2011.139

3. Seeman, N. C. Trends Biotechnol. 1999, 17, 437. doi:10.1016/S0167-7799(99)01360-8

4. Misra, R.; Acharya, S.; Sahoo, S. K. Drug Discovery Today 2010, 15, 842-850. doi:10.1016/j.drudis.2010.08.006

5. Mitra, S. B.; Wu, D.; Holmes, B. N. J. A. Dent. Assoc., JADA 2003, 134, 1382-1390. doi:10.14219/jada.archive.2003.0054

6. Lin, J. M.; Lin, H. Y.; Cheng, C. L.; Chen, Y. F. Nanotechnology 2006, 17, 4391. doi:10.1088/0957-4484/17/17/017

7. Herricks, T.; Chen, J.; Xia, Y. Nano Lett. 2004, 4, 2367. doi:10.1021/nl048570a

8. Kim, S. J.; Park, Y. J.; Ra, E. J.; Kim, K. K.; An, K. H.; Lee, Y. H.; Choi, J. Y.; Park, C. H.; Doo, S. K.; Park, M. H.; Yang, C. W. Appl. Phys. Lett. 2007, 90, 023114. doi:10.1063/1.2430993

9. Jin, R.; Cao, Y. C.; Hao, E.; Métraux, G. S.; Schatz, G. C.; Mirkin, C. A. Nature 2003, 425, 487. doi:10.1038/nature02020

10. Kittelson, D. B. J. Aerosol Sci. 1998, 29, 575-588. doi:10.1016/S0021-8502(97)10037-4

11. Joo, S. H.; Choi, S. J.; Oh, I.; Kwak, J.; Liu, Z.; Terasaki, O.; Ryoo, R. Nature 2001, 412, 169. doi:10.1038/35084046

12. White, C. W.; Withrow, S. P.; Sorge, K. D.; Meldrum, A.; Budai, J. D.; Thompson, J. R.; Boatner, L. A. J. Appl. Phys. 2003, 93, 5656. doi:10.1063/1.1565691

13. White, C. W.; Budai, J. D.; Zhu, J. G.; Withrow, S. P.; Aziz, M. J. Appl. Phys. Lett. 1996, 68, 2389. doi:10.1063/1.116143

14. Kumar, P.; Kumar, R.; Kanjilal, D.; Knobel, M.; Thakur, P.; Chae, K. H. J. Vac. Sci. Technol. B 2008, 26, L36-40. doi:10.1116/1.2956624

15. Avasthi, D. K.; Pivin, J. C. Curr. Sci. 2010, 98, 780-792.

16. Meldrum, A.; Boatner, L. A.; White, C. W.; Ewing, R. C. Mater. Res. Innovations 2000, 3, 190-204. doi:10.1007/s100190050003 
17. Xiang, X.; Zu, X. T.; Zhu, S.; Wei, Q. M.; Zhang, C. F.; Sun, K.; Wang, L. M. Nanotechnology 2006, 17, 2636. doi:10.1088/0957-4484/17/10/032

18. Kumar, P.; Rodrigues, G.; Lakshmy, P. S.; Kanjilal, D.; Singh, B. P.; Kumar, R. Nucl. Instrum. Methods Phys. Res., Sect. B 2006, 252, 354-360. doi:10.1016/j.nimb.2006.07.026

19. Kumar, P.; Rodrigues, G.; Lakshmy, P. S.; Kanjilal, D.; Kumar, R. J. Vac. Sci. Technol., A 2008, 26, 97. doi:10.1116/1.2823486

20. Prakash, J.; Tripathi, A.; Rigato, V.; Pivin, J. C.; Tripathi, J.; Chae, K. H.; Gautam, S.; Kumar, P.; Asokan, K.; Avasthi, D. K. J. Phys. D: Appl. Phys. 2011, 44, 125302. doi:10.1088/0022-3727/44/12/125302

21. Klimmer, A.; Ziemann, P.; Biskupek, J.; Kaiser, U.; Flesch, M. Phys. Rev. B 2009, 79, 155427. doi:10.1103/PhysRevB.79.155427

22. Hu, X.; Cahill, D. G.; Averback, R. S. J. Appl. Phys. 2002, 92, 3995 doi:10.1063/1.1503387

23. Khan, S. A.; Srivastava, S. K.; Avasthi, D. K. J. Phys. D: Appl. Phys. 2012, 45, 375304. doi:10.1088/0022-3727/45/37/375304

24. Singh, U. B.; Agarwal, D. C.; Khan, S. A.; Mohapatra, S.; Amekura, H.; Datta, D. P.; Kumar, A.; Choudhury, R. K.; Chan, T. K.; Osipowicz, T.; Avasthi, D. K. Beilstein J. Nanotechnol. 2014, 5, 105-110. doi:10.3762/bjnano.5.10

25. Singh, U. B.; Agarwal, D. C.; Khan, S. A.; Tripathi, A.; Kumar, A.; Choudhury, R. K.; Panigrahi, B. K.; Avasthi, D. K. Radiat. Eff. Defects Solids 2011, 166, 553-557. doi:10.1080/10420150.2011.572282

26. Singh, U. B.; Agarwal, D. C.; Khan, S. A.; Mohapatra, S.; Tripathi, A.; Avasthi, D. K. J. Phys. D: Appl. Phys. 2012, 45, 445304. doi:10.1088/0022-3727/45/44/445304

27. Padovani, S.; Scheurer, F.; Bucher, J. P. EPL 1999, 45, 327. doi:10.1209/epl/i1999-00167-2

28. Attri, A.; Nair, L. e-J. Surf. Sci. Nanotechnology 2013, 11, 99-104. doi:10.1380/ejssnt.2013.99

29. Szenes, G. Phys. Rev. B 1995, 51, 8026. doi:10.1103/PhysRevB.51.8026

30. Leuser, D.; Dunlop, A. Radiat. Eff. Defects Solids 1993, 126, 163. doi:10.1080/10420159308219701

31. Bolse, W. Nucl. Instrum. Methods Phys. Res., Sect. B 1993, 80-81, 137-141. doi:10.1016/0168-583X(93)96093-R

32. Ahmadi, T. S.; Wang, Z. L.; Green, T. C.; Henglein, A.; El-Sayed, M. A. Science 1996, 272, 1924-1925. doi:10.1126/science.272.5270.1924

33. Li, Y.; Gao, W.; Ci, L.; Wang, C.; Ajayan, P. M. Carbon 2010, 48, 1124. doi:10.1016/j.carbon.2009.11.034

34. Hrapovic, S.; Liu, Y.; Male, K. B.; Luong, J. H. Anal. Chem. 2004, 76, 1083. doi:10.1021/ac035143t

35. Si, Y.; Samulski, E. T. Chem. Mater. 2008, 20, 6792. doi:10.1021/cm801356a

36. Lu, L.; Sun, G.; Zhang, H.; Wang, H.; Xi, S.; Hu, J.; Tian, Z.; Chen, R. J. Mater. Chem. 2004, 14, 1005. doi:10.1039/b314868h

37. Kumar, P.; Rodrigues, G.; Rao, U. K.; Safvan, C. P.; Kanjilal, D.; Roy, A. Pramana 2002, 59, 805. doi:10.1007/s12043-002-0094-4

38. Ziegler, J. F.; Biersack, J. P.; Littmark, U. The Stopping and Range of lons in Solids; Pergamon Press: New York, 1996.

39. Ghatak, J.; Sundaravel, B.; Nair, K. G. M.; Satyam, P. V. J. Phys.: Condens. Matter 2008, 20, 485008. doi:10.1088/0953-8984/20/48/485008

40. Coffa, S.; Poate, J. M.; Jacobson, D. C. Phys. Rev. B 1992, 45, 8355. doi:10.1103/PhysRevB.45.8355

41. Holm, B.; Nielsen, K. B. J. Appl. Phys. 1995, 78, 5970. doi:10.1063/1.360600
42. Frank, F. C.; Turnbull, D. Phys. Rev. 1956, 104, 617. doi:10.1103/PhysRev.104.617

43. Simpson, P. J.; Szpala, S. J. Appl. Phys. 2002, 92, 5852. doi:10.1063/1.1516265

44. Caron, M.; Rothard, H.; Toulemonde, M.; Gervais, B.; Beuve, M. Nucl. Instrum. Methods Phys. Res., Sect. B 2006, 245, 36-40. doi:10.1016/j.nimb.2005.11.061

45. Rizza, G. C.; Strobel, M.; Heinig, K. H.; Bernas, H. Nucl. Instrum. Methods Phys. Res., Sect. B 2001, 178, 78-83. doi:10.1016/S0168-583X(01)00496-7

46. Zeng, H. C. Curr. Nanosci. 2007, 3, 177-181. doi:10.2174/157341307780619279

47. Powder Diffraction File, JCPDS International Center for Powder Diffraction Data: Pt (04-0802).

\section{License and Terms}

This is an Open Access article under the terms of the Creative Commons Attribution License

(http://creativecommons.org/licenses/by/2.0), which permits unrestricted use, distribution, and reproduction in any medium, provided the original work is properly cited.

The license is subject to the Beilstein Journal of

Nanotechnology terms and conditions:

(http://www.beilstein-journals.org/bjnano)

The definitive version of this article is the electronic one which can be found at: doi:10.3762/bjnano.5.197 